\title{
Spectral Dependence of Transmission Losses in High-Index Polymer Coated no-Core Fibers
}

\author{
Xiaokang Lian \\ Technological University Dublin \\ Gerald Farrell \\ Technological University Dublin, gerald.farrell@tudublin.ie \\ Qiang Wu \\ Northumbria University
}

See next page for additional authors

Follow this and additional works at: https://arrow.tudublin.ie/engscheleart2

Part of the Biomedical Engineering and Bioengineering Commons

\section{Recommended Citation}

Lian, X. et al. (2020) Spectral Dependence of Transmission Losses in High-Index Polymer Coated no-Core Fibers, Journal of Lightwave Technology · July 2020 DOI: 10.1109/JLT.2020.3010101

This Article is brought to you for free and open access by the School of Electrical and Electronic Engineering at ARROW@TU Dublin. It has been accepted for inclusion in Articles by an authorized administrator of ARROW@TU Dublin. For more information, please contact arrow.admin@tudublin.ie, aisling.coyne@tudublin.ie, gerard.connolly@tudublin.ie.

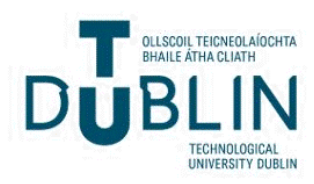


Authors

Xiaokang Lian, Gerald Farrell, Qiang Wu, Wei Han, Changyu Shen, Youqiao Ma, and Yuliya Semenova

This article is available at ARROW@TU Dublin: https://arrow.tudublin.ie/engscheleart2/257 
See discussions, stats, and author profiles for this publication at: https://www.researchgate.net/publication/343038632

\section{Spectral dependence of transmission losses in high-index polymer coated no- core fibers}

Article in Journal of Lightwave Technology · July 2020

DOI: 10.1109/JLT.2020.3010101

CITATION

7 authors, including:

0

Xiaokang Lian

Technological University Dublin - City Campus

17 PUBLICATIONS 101 CITATIONS

SEE PROFILE

(2) Qiang Wu

Northumbria University

328 PUBLICATIONS 3,502 CITATIONS

SEE PROFILE
READS

226

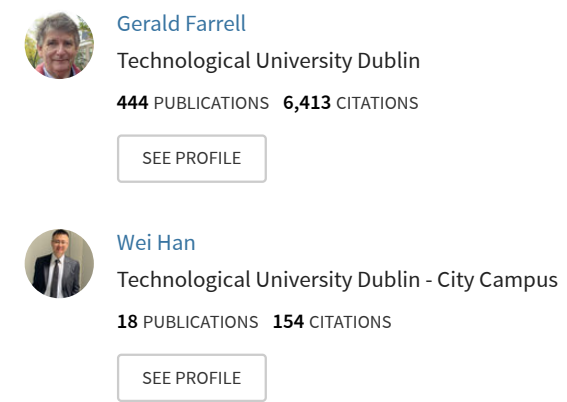

Some of the authors of this publication are also working on these related projects:

12th IEEE-IET Intern. Symposium on COMMUNICATION SYSTEMS, NETWORKS AND DIGITAL SIGNAL PROCESSING -20-22 July 2020, Porto, PORTUGAL

[https://csndsp2020.av.it.pt/] View project

Directly pumped Ho3+-doped microspheres lasing at 2.0 $\mu \mathrm{m}$ View project 


\title{
Spectral dependence of transmission losses in high-index polymer coated no-core fibers
}

\author{
Xiaokang Lian, Gerald Farrell, Qiang Wu, Wei Han, Changyu Shen, Youqiao Ma, and Yuliya \\ Semenova.
}

\begin{abstract}
A high-index polymer coated no-core fiber (PC-NCF) is effectively a depressed core fiber, where the light is guided by the anti-resonant, inhibited coupling and total internal reflection effects and the dispersion diagram shows periodic resonant and anti-resonant bands. In this paper, the transmission spectra of the straight and bent PC-NCFs (length $>5 \mathrm{~cm}$ ) are measured and analyzed from a modal dispersion perspective. For the purpose of the study, the PC-NCFs are contained within a fiber hetero-structure using two single-mode fiber (SMF) pigtails forming a SMF-PC-NCF-SMF structure. The anti-resonant spectral characteristics are suppressed by the multimode interference in the PC-NCF with a short fiber length. The increase of the length or fiber bending (bend radius $>28 \mathrm{~cm}$ ) can make the anti-resonance dominate and result in the periodic transmission loss dips and variations in the depth of these loss dips, due to the different modal intensity distributions in different bands and the material absorption of the polymer. The PC-NCFs are expected to be used in many devices including curvature sensors and tunable loss filters, as the experiments show that the change of loss dip around $1550 \mathrm{~nm}$ is over $31 \mathrm{~dB}$ and the average sensitivity is up to $14.77 \mathrm{~dB} / \mathrm{m}^{-1}$ in the bend radius range from $\infty$ to $47.48 \mathrm{~cm}$. Our study details the general principles of the effect of high-index layers in the formation of the transmission loss dips in fiber optics.
\end{abstract}

Index Terms-Optical fibers, Optical fiber devices, Optical fiber losses, Optical fiber dispersion, Optical fiber interference.

\section{INTRODUCTION}

$\mathrm{C}$ OATING of optical fibers has been extensively studied as a key element of the design of various devices such as

This Manuscript received XX XX, 2020; revised XX XX, 2020; accepted XX XX, 2020. Date of publication XX XX, 2020; date of current version XX XX, 2020. This work was supported by the Technological University Dublin under Fiosraigh scholarship program. (Corresponding author: Xiaokang Lian.)

X. Lian, G. Farrell, W. Han and Y. Semenova are with the Photonics Research Center, Technological University Dublin, Dublin D08 X622, Ireland (e-mail: d15128010@mytudublin.ie; gerald.farrell@tudublin.ie; d14125980@mytudublin.ie; yuliya.semenova@tudublin.ie).

Q. Wu is with the Department of Mathematics, Physics, and Electrical Engineering, Northumbria University, Newcastle upon Tyne NE1 8ST, U.K., and also with the Key Laboratory of Nondestructive Test (Ministry of Education) of Nanchang Hangkong University, Nanchang 330063, China (e-mail: qiang.wu@northumbria.ac.uk).

C. Shen is with the Institute of Optoelectronic Technology, China Jiliang University, Hangzhou 310018, China (e-mail: shenchangyu@cjlu.edu.cn).

$\mathrm{Y}$. Ma is with the Department of Electrical and Computer Engineering, Dalhousie University, Halifax B3H 4R2, Canada (e-mail: mayouqiao188@hotmail.com).

Color versions of one or more of the figures in this paper are available online at http://ieeexplore.ieee.org.

Digital Object Identifier 10.1109/JLT.2020.2784678 XXX optical sensors [1-10], filters [11,12] and modulators [13]. Fiber coating materials include metals, metal oxides, graphene, polymers, and many others. These materials are either chosen to be sensitive to specific physical, chemical and bioparameters in order to enhance the sensitivity of the sensor [14-19] or to facilitate particular waveguiding mechanisms $[2,3,20]$. For example, thin films of silver or gold deposited on an optical fiber can form surface plasmon resonances due to the coupling between light and the surface electrons of the metal films [2]. Lossy films (i.e., indium-tin oxide (ITO) [20]) on the surface of no-core fibers (NCFs) can cause lossy mode resonances (LMRs), due to the coupling between the guided modes in the optical fiber and the lossy modes in the fiber coating [3].

The high refractive index coated fibers show exceptional modal characteristics. The high-index coating can cause mode reorganization [14-16], when a core mode in the fiber is coupled into the modes of the high-index coating and other fiber modes are reorganized. The mode reorganization phenomenon along with drastic changes of the modal field distributions were also observed numerically in a LMR fiber structure [21,22], where the real part of the complex refractive index of the lossy coating is higher than that of the fiber and the surrounding medium. The energy of the lossy modes was demonstrated numerically to be distributed both in the fiber core and the lossy coating along with a mode splitting phenomenon near the resonant wavelength [23]. Although a number of methods have been employed to investigate the high-index coated fiber structure [14-23], it was rarely mentioned that a high-index coating changes the index distribution within a fiber. For example, an NCF is a typical conventional step-index fiber, which consists of a silica core surrounded by the low-index air acting as its cladding. Light guiding within the NCF is based on the total internal reflection effect. If an NCF is coated with a high-index material, its original index distribution changes to the inversed index distribution with the high-index coating acting as the new fiber cladding, and the light guiding mechanism in such a fiber is also changed. This inversed-index structure can be considered as a depressed core fiber [24].

In our previous paper [25], a dispersion diagram of the depressed core fiber was obtained by solving the full-vector eigenvalue equations. Analysis of the dispersion diagram suggests that the light guiding mechanism in the fiber core is supported by the anti-resonant, inhibited coupling and total 
internal reflection effects. The anti-resonant effect is typically described by an anti-resonant reflecting optical waveguide (ARROW) model [26]. In this model, the high-index cladding region of the depressed core fiber is treated as a Fabry-Perot resonator, which allows the anti-resonant light to be reflected back while allowing forward transmission of the resonant light. The inhibited coupling mechanism explains the coexistence of cladding- and core-type modes in the same effective refractive index- wavelength $\left(n_{\text {eff }}-\lambda\right)$ dispersion space. To date the anti-resonant and inhibited coupling guiding nature of the high refractive index coated fiber structure, has not been exploited.

In this paper, a high-index polymer coated no-core fiber (PC-NCF) is studied, which consists of an NCF with acrylate polymer coating. Compared to a semiconductor coating with a complex refractive index in the LMR fiber structure [20], the refractive index of the acrylate polymer is a real number [27-29]. The PC-NCF can be used as a model for studies of the waveguiding properties of the depressed core fiber, since the refractive index of the polymer coating (as a cladding region) is higher than that of the silica core. In section III, the spectra of the straight and bent PC-NCFs with several different lengths were measured and periodic transmission loss dips were found in the spectra of straight PC-NCFs with long lengths and in bent PC-NCFs. The effects of the multimode interference and the anti-resonance on the spectral characteristics of the PC-NCFs are analyzed. With the knowledge of the anti-resonant and inhibited coupling guiding mechanisms, the relationship between the modal field distribution and the transmission loss is studied. In section IV, the implications of the results for the LMR fiber structures are discussed.

\section{METHODS}

The PC-NCF used in this paper is FG125LA from Thorlabs, which is an NCF coated with a layer of acrylate polymer. The microscopic image and the cross-sectional view of the PC-NCF are shown in Fig. 1(a) and Fig. 1(b), respectively. The PC-NCF is described by a three-layer fiber model, where the silica NCF plays the role of a core region, the polymer coating serves as the cladding region and the surrounding medium air acting in effect as a second cladding region. The refractive indices of the three layers are $n_{1}, n_{2}$ and $n_{3}$ respectively, with $n_{2}>n_{1}>n_{3}$. The transmission spectra of the straight and bent PC-NCFs are studied, where the PC-NCF is spliced with two single mode fiber (SMF) pigtails forming a fiber hetero-structure SMF-PC-NCF-SMF as shown in Fig. 4. The experimental setup is shown in Fig. 1(c), where the fiber is fixed on two translation stages and the bending curvature of the PC-NCF is controlled by moving the two stages toward one another. The bend radius is calculated based on the displacement of the translation stages [30].

In our previous work [25] the effective refractive indices and the transverse intensity profiles of modes in a depressed core fiber were calculated by solving the full-vector eigenvalue equations, with the fiber parameters the same as those of the studied PC-NCF. In this paper the calculations for both the straight and bent PC-NCFs were carried out by the full-vector (a)

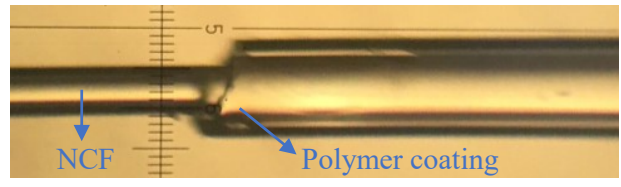

(b)

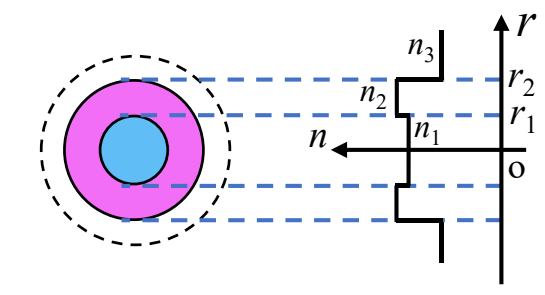

(c)

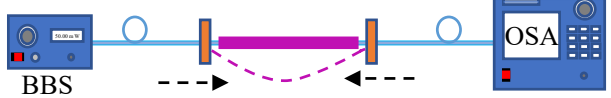

Fig. 1. (a) Microscopic image of the polymer coated no-core fiber (PC-NCF). (b) Refractive index variations along the cross section of the PC-NCF $\left(n_{2}>n_{1}>\right.$ $n_{3}$ ). (c) Schematic of the experimental setup for measurements of the straight and bent PC-NCF samples.

finite element method with COMSOL Multiphysics, where the maximum mesh size is $0.4 \lambda$ and the relative error in the computed eigenvalues is smaller than $10^{-12}$. The numerical results obtained agree well with the analytical results in [25] for the straight PC-NCF. Furthermore, the waveguiding in the straight PC-NCF was analyzed using the ray optics approach and the transmission loss spectra were simulated analytically based on an asymmetric planar reflection model.

In the calculations, the refractive indices of the silica NCF and the polymer coating are assumed as $n_{1}=1.445$ and $n_{2}=$ 1.51 at $\lambda=1550 \mathrm{~nm}$, respectively. The radii of the silica NCF and the polymer coating are $r_{1}=62.5 \mu \mathrm{m}$ and $r_{2}=125 \mu \mathrm{m}$ respectively. The thickness of the polymer coating is $d=62.5$ $\mu \mathrm{m}$. The surrounding medium is air with the refractive index of $n_{3}=1$. The radii and the refractive indices of the core (co) and cladding (cl) regions of the input/output SMF (from Corning) are $r_{\mathrm{co}}=4.15 \mu \mathrm{m}, r_{\mathrm{cl}}=62.5 \mu \mathrm{m}, n_{\mathrm{co}}=1.4504$ and $n_{\mathrm{cl}}=1.4447$. The material dispersion was not considered in the calculations for the sake of conciseness.

In the experiments, a broadband light source (Thorlabs S5FC1005s, $1030 \mathrm{~nm}-1660 \mathrm{~nm}$ ) and an optical spectrum analyzer (OSA, Agilent 86142B) were used. The polymer coating with the length of about $0.5 \mathrm{~cm}$ at both ends of the PC-NCF was removed before splicing with the SMFs, as shown in Fig. 1(a). Any slight unevenness at the end points of the remaining polymer coating does not affect the experimental results for the transmission spectral loss. The ends of the bare NCF and the SMF have the same diameter as shown in Fig. 4 and were axially aligned and spliced using a Fujikura $70 \mathrm{~S}$ splicer in automatic mode.

\section{RESULTS}

\section{A. Modes in the straight PC-NCFs}

The modes in PC-NCFs include $\mathrm{TE}_{0, \mathrm{~N}}, \mathrm{TM}_{0, \mathrm{~N}}, \mathrm{HE}_{\mathrm{m}, \mathrm{N}}$ and $\mathrm{EH}_{\mathrm{m}, \mathrm{N}}$, where ' 0 ' and ' $\mathrm{m}$ ' on the left side of the comma in the subscript positions are the azimuthal mode numbers and ' $\mathrm{N}$ ' on 
the right side are the radial mode numbers. Fig. 2(a) shows the dispersion diagram $\left(n_{\text {eff }} \mathrm{vs.} \lambda\right)$ for the modes $\mathrm{HE}_{1, \mathrm{~N}}, \mathrm{~N}=34,35$, ..., 38. The cladding modes, which are guided in the cladding region by total internal reflections are depicted within the top part of the graph where $n_{\text {eff }}>n_{1}$. On a same dispersion curve, as $\lambda$ increase the cladding modes $\mathrm{HE}_{1, \mathrm{~N}}$ can change into anti-resonant core modes $\mathrm{HE}_{1, \mathrm{n}+(\mathrm{N}-\mathrm{n})}, \mathrm{n}=1,2, \ldots$, where the subscript ' $n$ ' denotes the radial number in the core region while the number ' $\mathrm{N}-\mathrm{n}$ ' denotes the radial number in the cladding region [25]. The total radial number does not change for each of the dispersion curves. The step-like dispersion curves consistent with the mode reorganization phenomenon [14-16]. The $\mathrm{HE}_{1, \mathrm{n}+(\mathrm{N}-\mathrm{n})}$ exhibit similar qualitative and quantitative behavior as the $\mathrm{HE}_{1, \mathrm{n}}$ modes of conventional step-index fibers and can be approximated as the linear polarization modes $\mathrm{LP}_{0, \mathrm{n}}$ in the NCF [25]. As shown in the Fig. 2(a), the dispersion curves of the modes $\mathrm{LP}_{0, \mathrm{n}}$ shown in orange color are overlapped with the $\mathrm{HE}_{1, \mathrm{n}+(\mathrm{N}-\mathrm{n})}$ in the PC-NCF.

Fig. 2(a) exhibits periodic strong and moderate index dispersion bands, corresponding to resonant and anti-resonant bands. The resonant bands are indicated by the vertical black and red dashed lines, which intersect with the horizontal purple dashed line corresponding to the value of $n_{1}$. The intersect points can be approximated as the cutoff positions of the $\mathrm{TM}_{\mathrm{N}}$ and $\mathrm{TE}_{\mathrm{N}}$ modes in the equivalent asymmetric planar waveguide, calculated by $[25,31]$ :

$$
\lambda_{\mathrm{N}, \mathrm{c}}=\frac{2 d \sqrt{n_{2}^{2}-n_{1}^{2}}}{\left[\mathrm{~N}-1+\frac{1}{\pi} \tan ^{-1}\left(\kappa \frac{\sqrt{n_{1}^{2}-n_{3}^{2}}}{\sqrt{n_{2}^{2}-n_{1}^{2}}}\right)\right]}, \kappa= \begin{cases}1, & \text { for } \mathrm{TE}_{\mathrm{N}} \text { modes } \\ \frac{n_{2}^{2}}{n_{3}^{2}} & \text { for } \mathrm{TM}_{\mathrm{N}} \text { modes }\end{cases}
$$

where, $\mathrm{N}=34,35, \ldots, 38$.

In order to analyze the modal field distributions in different dispersion bands, one dispersion period indicated by the red dashed rectangle in Fig. 2(a) is enlarged and shown in Fig. 2(b). The modal intensity and electric field vector distributions at several points indicated in Fig. 2(b) are shown in Fig. 3. It should be noted that the HE mode is a two-fold degeneracy mode, including the modes $\mathrm{HE}_{\mathrm{a}}$ and $\mathrm{HE}_{\mathrm{b}}$, whose modal intensity distributions are identical except for a $\pi / 2$ rotation of the electric vector as shown later in Fig. 8. Fig. 3 shows only one of the two degenerate modes for simplicity. For the cladding mode $\mathrm{HE}_{1,36}$ at point $\mathrm{A}$ the modal energy is mainly confined in the fiber cladding region, for the anti-resonant core mode $\mathrm{HE}_{1,1+(35)}$ at point $\mathrm{D}$ the modal energy is mainly confined in the fiber core region. The modes at $\mathrm{B}$ and $\mathrm{C}$ are denoted as $\left[\mathrm{HE}_{1,1+(35)}\right]$, where the brackets indicate that the modes are in a transition state and their modal energy is evenly distributed both in the fiber core and cladding regions. The modes at points $\mathrm{E}$ and $\mathrm{F}$ are also in transition states and denoted as $\left[\mathrm{HE}_{1,1+(35)}\right]$ or $\left[\mathrm{HE}_{1,2+(34)}\right]$. Similarly, the modes at points A' and D' represent the low-order and high-order anti-resonant core modes $\mathrm{HE}_{1,1+(36)}$ and $\mathrm{HE}_{1,2+(35)}$, respectively. The modes at points $\mathrm{B}^{\prime}$, $\mathrm{C}^{\prime}, \mathrm{E}^{\prime}$ and $\mathrm{F}^{\prime}$ are in transition states.

Another modal characteristic of the PC-NCF is the coexistence of the core and cladding modes in the dispersion
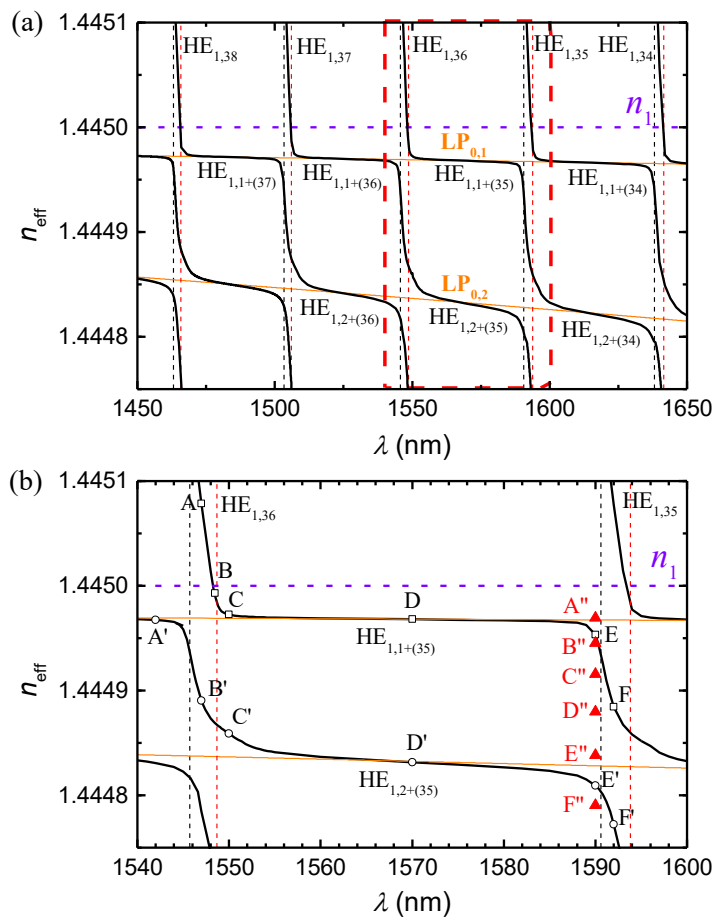

Fig. 2. (a) shows the dispersion curves ( $n_{\text {eff }}$ vs. $\lambda$ ) of modes $\mathrm{HE}_{1, \mathrm{~N}}, \mathrm{~N}=34,35$, $\ldots, 38$ in the PC-NCF, which change into the anti-resonant core modes $\mathrm{HE}_{1, \mathrm{n}+(\mathrm{N}-\mathrm{n})}, \mathrm{n}=1,2, \ldots$ as $\lambda$ increases. The orange lines are the dispersion curves of the $\mathrm{LP}_{0,1}\left(\mathrm{HE}_{1,1}\right)$ and $\mathrm{LP}_{0,2}\left(\mathrm{HE}_{1,2}\right)$ in the NCF. (b) is the partially enlarged image of (a), indicated by the red dashed frame. The black and red vertical dashed lines indicate the resonant bands.

(a)

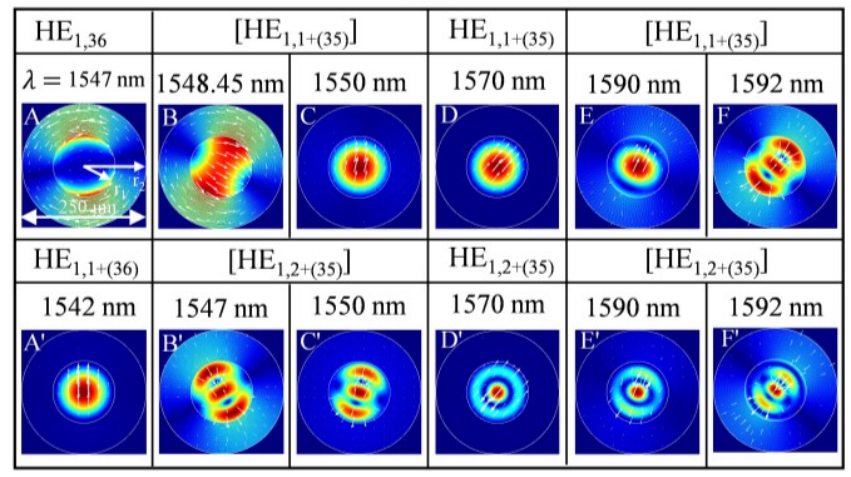

(b)

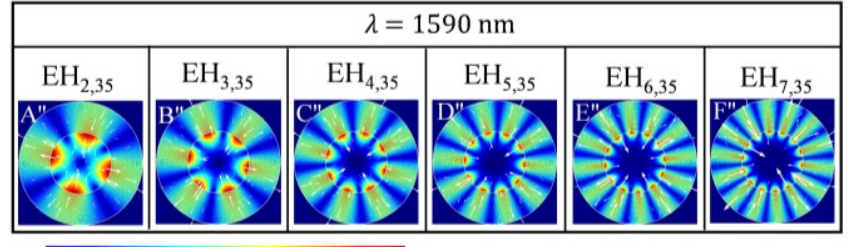

$0 \longdiv { 1 }$

Fig. 3. The intensity and electric field vector distributions of modes in PC-NCF, whose positions in dispersion diagram are indicated in Fig. 2(b).

space [25]. Some cladding modes exist in the dispersion space below the horizontal purple dashed line corresponding to the value of $n_{1}$. For example, the modes $\mathrm{EH}_{\mathrm{m}, 35}, \mathrm{~m}=2,3, \ldots 7$ at points A"- F" with the wavelength of $1590 \mathrm{~nm}$, whose modal intensities and electric field vector distributions are shown in 
Fig. 3(b). Although the energy of these cladding modes at points A"- F" with $n_{\text {eff }}<n_{1}$ is mainly confined in the cladding region, they differ from the cladding modes with $n_{\text {eff }}>n_{1}$, since the total internal reflection does not apply at the inner boundary of the cladding region. The confinement of energy in the cladding region for the modes at points A"- F" is attributed to the anti-resonant and inhibited coupling effects.

If the material dispersion is considered, the refractive index of the polymer coating will decrease smoothly as the wavelength increases according to the Cauchy-Schott equation [27]. Based on Eq. (1), the resonant coupling position is mainly dependent on the thickness and the refractive index of the coating. Therefore, the anti-resonance condition is maintained if an inversed index profile (the refractive index of the cladding is higher than that of the fiber core) is maintained. The changes of the index due to material dispersion will only modify the resonant wavelength positions (period), which may cause the discrepancies between the simulated and measured results, as discussed in section III-C. If the inversed index profile is unchanged, then the modal field distributions in both the resonant and anti-resonant bands will not be affected by changes in the refractive index of the polymer coating.

The process of mode coupling in the LMR and PC-NCF structures is different, which depends on the overlap of modal fields and the phase-matching conditions (equality of real parts of the effective refractive index). As shown in the dispersion diagram and the mode profiles for the PC-NCF in Figs. 2 and 3 and Ref. [25], the coupling between the cladding modes and the anti-resonant core modes takes place in accordance with the order of the modes and the symmetry of the modal field distributions, that is the fundamental core modes are at first coupled to the corresponding cladding modes and then the high-order core modes become low-order core modes due to mode reorganization. The field of the core modes in both fiber structures is distributed throughout the entire fiber cross section. However, the parts of modal field of the core modes which propagate in the high-loss coating layer of the LMR structure may be absorbed and changed to a different extent based on their different symmetries. As a result, in the LMR structure mode coupling occurs in a less predictable manner and some high-order modes may couple to cladding modes before the low-order modes, as shown in the Refs. [21] and [22].

\section{B. Analysis of the effects of anti-resonance and multimode interference on the transmission spectra of the PC-NCFs}

The complex modal dispersion indicates the actual light field in the PC-NCF is very complex, possessing both anti-resonance (AR) and the multimode interference (MMI) properties. Fig. 4 shows a ray trajectory within the SMF-PC-NCF-SMF hetero-structure. The light in the lead-in SMF excites multiple $\mathrm{LP}_{0, \mathrm{n}}\left(\mathrm{HE}_{1, \mathrm{n}}\right)$ modes in the NCF. An individual guided $\mathrm{LP}_{0, \mathrm{n}}$ mode indicated by the red arrows in the NCF will progressively reflect between the polymer coatings at a certain angle $\theta_{1}$, forming the multiple reflections and multi-path interference at the inner boundary of the polymer coating region, which corresponds to the anti-resonance property. All the black and

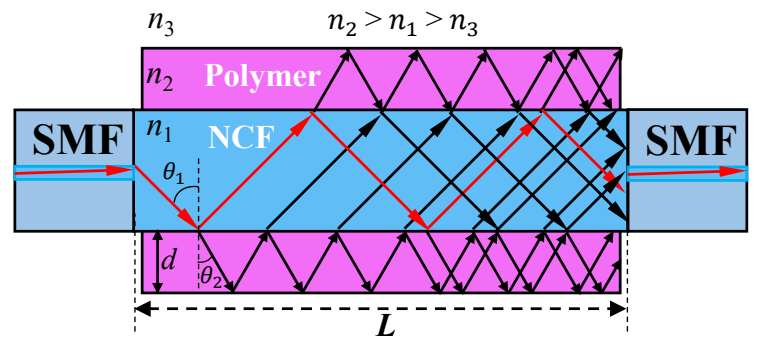

Fig. 4. Schematic of the ray trajectories of an excited guide mode within the SMF-PC-NCF-SMF hetero-structure.

red arrows in the middle section fiber represent the ray trajectories of an guided mode $\mathrm{HE}_{1, \mathrm{n}+(\mathrm{N}-\mathrm{n})}$ in the PC-NCF. After the propagation within the PC-NCF light will couple into the lead-out SMF. The transmitted power in the lead-out SMF is dependent on the coupling between its modal field and the field in the silica NCF.

The $n_{\text {eff }}\left(\mathrm{LP}_{0, \mathrm{n}}\right)$ can be written as $[31,32]$ :

$$
n_{\mathrm{eff}}\left(\mathrm{LP}_{0, \mathrm{n}}\right)=\frac{1}{k_{0}} \sqrt{k_{0}^{2} n_{1}^{2}-\frac{1}{r_{1}^{2}}\left[\left(2 \mathrm{n}-\frac{1}{2}\right) \frac{\pi}{2}\right]^{2}}
$$

where $k_{0}=2 \pi / \lambda$ is wavenumber in vacuum. The angle $\theta_{0, \mathrm{n}}$ (equal to $\theta_{1}$ in Fig. 4) between the incident direction of the mode $\mathrm{LP}_{0, \mathrm{n}}$ (at the boundary) and the radial direction can be calculated with $n_{1} \sin \theta_{0, \mathrm{n}}=n_{\mathrm{eff}}\left(\mathrm{LP}_{0, \mathrm{n}}\right)$, as:

$$
\theta_{0, \mathrm{n}}=\sin ^{-1}\left(\frac{n_{\mathrm{eff}}(\mathrm{LP} 0, \mathrm{n})}{n_{1}}\right)
$$

The energy coupling coefficient $\eta_{0, \mathrm{n}}$ between the fundamental mode in the SMF and the guided mode $\mathrm{LP}_{0, \mathrm{n}}$ in the NCF can be calculated as [32-34]:

$$
\eta_{0, \mathrm{n}}=\frac{\left|\int_{0}^{\infty} E_{\mathrm{S}}(r) \mathrm{E}_{0, \mathrm{n}}(r) r d r\right|^{2}}{\int_{0}^{\infty}\left|E_{\mathbf{S}}(r)\right|^{2} r d r \int_{0}^{\infty}\left|E_{0, \mathrm{n}}(r)\right|^{2} r d r}
$$

where $E_{\mathrm{S}}$ and $E_{0, \mathrm{n}}$ represent the field profiles of the fundamental mode in the SMF and the $\mathrm{LP}_{0, \mathrm{n}}$ mode in the NCF, respectively.

The multiple reflections of a ray $\left(\mathrm{LP}_{0, \mathrm{n}}\right)$ from the high-index polymer coating of the PC-NCF can be approximated as the multiple reflections of an unpolarized incident beam on an equivalent asymmetric planar waveguide as shown in the Appendix. The ratio of reflection intensity $(\bar{R})$ of the unpolarized incident beam is calculated by Eq. (19). Using Eqs. (2)-(4) and (19), the transmission efficiency of the hetero-structure SMF-PC-NCF-SMF induced by the multiple reflections (the AR effect) can be calculated as:

$$
I_{\mathrm{AR}}=10 \log _{10}\left(\sum \eta_{0, \mathrm{n}}^{2} \bar{R}_{0, \mathrm{n}}\right)
$$

where $\bar{R}_{0, \mathrm{n}}$ is corresponding to the mode $\mathrm{LP}_{0, \mathrm{n}}$. Eq. (5) is a scalar superposition of the transmission efficiency of all individual modes $\mathrm{HE}_{1, \mathrm{n}+(\mathrm{N}-\mathrm{n})}$ in the PC-NCF. 
In Eq. (5), the interference between different guided modes is not considered, which is a superposition of both the intensity and the phase of different guided modes $\mathrm{HE}_{1, \mathrm{n}+(\mathrm{N}-\mathrm{n})}$ in the PC-NCF. The interference between different guided modes $\mathrm{HE}_{1, \mathrm{n}+(\mathrm{N}-\mathrm{n})}$ in the $\mathrm{PC}-\mathrm{NCF}$ is similar to and can be approximated as the multimode interference of $\mathrm{LP}_{0, \mathrm{n}}\left(\mathrm{HE}_{1, \mathrm{n}}\right)$ modes in an SMF-NCF-SMF [32-34], since the $n_{\text {eff }}$ and the mode field distribution of $\mathrm{HE}_{1, \mathrm{n}+(\mathrm{N}-\mathrm{n})}$ modes in $\mathrm{PC}-\mathrm{NCF}$ can be approximated by that of the $\mathrm{LP}_{0, \mathrm{n}}\left(\mathrm{HE}_{1, \mathrm{n}}\right)$ modes in the NCF as discussed in section III-A and Ref. [25].

The transmission efficiency of the straight SMF-NCF-SMF based on the MMI effect can be calculated by [33]:

$$
I_{\mathrm{MMI}}=10 \log _{10}\left(\left|\sum \eta_{0, \mathrm{n}} \exp \left(i \frac{2 \pi n_{\mathrm{eff}}\left(\mathrm{LP}_{0, \mathrm{n}}\right) L}{\lambda}\right)\right|^{2}\right)
$$

which is related to the length $L$. Combining Eqs. (5) and (6), the transmission efficiency of the SMF-PC-NCF-SMF including both the $\mathrm{AR}$ and the MMI effects can be calculated as:

$$
I=10 \log _{10}\left(A \sum \eta_{0, \mathrm{n}}^{2} \bar{R}_{0, \mathrm{n}}+B\left|\sum \eta_{0, \mathrm{n}} \exp \left(i \frac{2 \pi n_{\mathrm{eff}}\left(\mathrm{LP}_{0, \mathrm{n}}\right) L}{\lambda}\right)\right|^{2}\right)
$$

where $\mathrm{A}$ and $\mathrm{B}(\mathrm{A}+\mathrm{B}=1)$ are defined as intensity coefficients for the AR and MMI effects, respectively. These coefficients are used to set the relative levels of AR interference and MMI for the purpose of discussion below.

Figs. 5(a)-5(d) show the simulated transmission spectra of the hetero-structure SMF-PC-NCF-SMF with $L=5.8 \mathrm{~cm}$ (approximately one self-imaging distance), calculated by Eq. (7). The spectrum in Fig. 5(a) calculated with $\mathrm{A}=0$ and $\mathrm{B}=1$ is as expected similar to the simulated and measured results for an SMF-NCF-SMF in our previous work Ref. [35], where the transmission peaks and dips are solely due to the MMI effect. As the intensity coefficient A increases to 0.5 in Fig. 5(b) and then to 1 in Fig. 5(d), the AR characteristics in the spectrum become more and more pronounced, showing periodic transmission dips aligned with the vertical black and red dashed lines. The results are similar for the hetero-structure SMF-PC-NCF-SMF with $L=75.8 \mathrm{~cm}$, as shown in Figs. 5(e)-5(h). It should be noted that the spectra for different values of $L$ in Figs. 5(d) and 5(h) are the same, indicating the AR effect ( $\mathrm{A}=1, \mathrm{~B}=0)$ is independent of the value of length $L$.

The depth of the transmission loss dip around $1550 \mathrm{~nm}$ in Fig. 5(d) and Fig. 5(h) is $1.97 \mathrm{~dB}$ in both cases, this is calculated as the difference between the minimum value of the transmission loss taken at the dip labelled $\mathrm{P} 3$ and the average of the two adjacent transmission levels at the points labelled P1 and P2, as shown in Fig. 5(d).

The above analysis indicates that the MMI effect can be suppressed to obtain a periodic transmission spectrum due to AR. This can be achieved either by increasing the length of the PC-NCF or by bending the fiber as discussed in the sections which follow below.
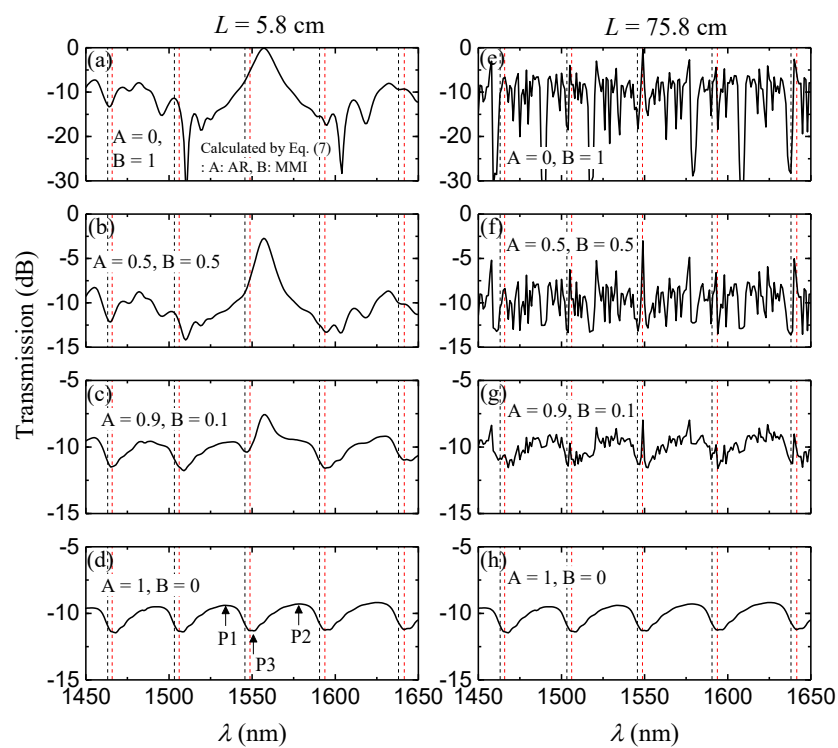

Fig. 5. The transmission spectra of the SMF-PC-NCF-SMF hetero-structure, calculated by Eq. (7) with different intensity coefficients $A$ and $B$ as indicated. (a)-(d) $L=5.8 \mathrm{~cm}$; (e)-(f) $L=75.8 \mathrm{~cm}$. The black and red dashed vertical lines indicate the resonant bands.

\section{Measured transmission spectra of straight PC-NCFs and the effect of PC-NCF length $L$}

Fig. 6 shows the experimentally recorded spectra for a straight SMF-PC-NCF-SMF with different values of $L$ increasing from $5.8 \mathrm{~cm}$ to $85.8 \mathrm{~cm}$. The spectra as a function of length in the range from $5.8 \mathrm{~cm}$ to $38 \mathrm{~cm}$ shown in Figs. 6(a)-6(c) display irregular shapes, where the differences are consistent with the presence of multimode interference. For the sample with a longer PC-NCF length $L=75.8 \mathrm{~cm}$ in Fig. 6(d), the transmission spectrum exhibits periodic transmission dips and windows, similar to those in the simulated spectrum shown in Figs. 5(d) and 5(h) in terms of the spectral positions of the transmission dips and their depth, consistent with AR interference. The slight difference in the spectral period of the simulated and experimental results is due to the material dispersion, which has not been considered in the simulation. The periodic spectral characteristics are maintained while the intensity of the transmission windows is reduced for the PC-NCF with a longer $L$ of $85.8 \mathrm{~cm}$ as shown in Fig. 6(e). The progressive increase of loss in the transmission window as the length increases is related to the modal attenuation due to the material absorption.

The experimental results imply that the multimode interference effect is suppressed in the hetero-structures with a sufficiently large $L$, which can be explained as follows. There are multiple modes in both the anti-resonant and resonant bands shown in Fig. 2(a), therefore in principle the multimode effects can occur in all the bands with the result that the spectrum is dependent on the length $L$. However, the field distributions of modes in different bands are different, as discussed in section III-A. The energy of anti-resonant core modes is mainly confined in the silica core region while that of the transition modes is distributed in both the silica core and the cladding regions. The modes propagating in the fiber suffer attenuation 


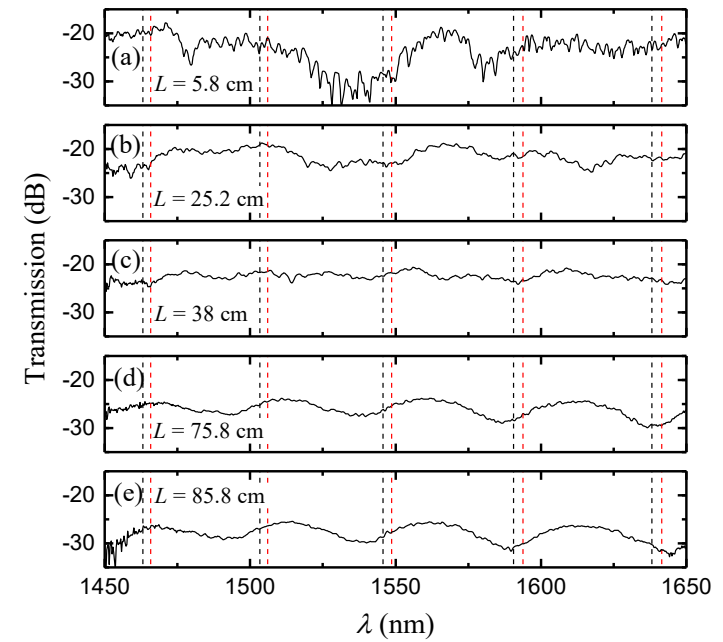

Fig. 6. Measured transmission spectra of the straight hetero-structures SMF-PC-NCF-SMF with different $L$. The black and red dashed vertical lines indicate the resonant bands.

due to the material absorption, which is significantly larger in the polymer cladding (with a propagation loss level of $0.6 \mathrm{~dB}$ $\mathrm{cm}^{-1}[35]$ ) than in the silica core (a loss level of $0.2 \times 10^{-5} \mathrm{~dB}$ $\mathrm{cm}^{-1}$ ). Therefore, the transition modes suffer greater attenuation as a function of distance than the anti-resonant core modes. The transition modes will fade earlier than the anti-resonant core modes during the propagation process, due to the increase of attenuation with the length $L$. For larger values of $L$, the higher attenuation of the transition modes compared to the anti-resonant core modes leads to the suppression of the multimode interference in the resonant bands and the appearance of periodic transmission dips, as shown in Figs. 6(d) and 6(e). For shorter values of $L$, the difference in the modal attenuation in the resonant and anti-resonant bands is minor, therefore the multimode property is present for both bands, which in turn is why the transmission spectrum in Figs. 6(a)-6(c) is dependent on the length $L$.

\section{Modal field distortions in the bent PC-NCFs}

In this section and the following section III-E the effect of bending induced mode field distortions on propagation and the relative strength of AR interference and MMI are considered. In the simulations, a bent PC-NCF is transformed to an equivalent, straight fiber by the process of conformal mapping [36]. The refractive index distribution in the equivalent straight PC-NCF is expressed as [36]:

$$
n_{i}^{\prime}(x, y)=n_{i}(x, y) \exp \left(\frac{x}{R}\right), i=1 \text { or } 2
$$

where $n_{i}(x, y)$ is the refractive index of the bent fiber cross section, which can be approximated as that of the straight fiber if the stress-optic effect is not considered. $x$ is a transverse coordinate, with its origin in the center of the fiber and a positive value indicating the magnitude of the distance along a line joining the center of curvature and the center of the fiber. $R$ is the bend radius. In the simulation of the bent fiber structure by the finite element method, a perfectly matched layer is set at the outer boundary of the polymer coating to absorb the possible outgoing energy. In practice, the energy loss from the outer boundary of the polymer coating can be neglected for the studied range $(R>28 \mathrm{~cm})$, since the imaginary part of $n_{\text {eff }}$ is $10^{10}$ times smaller than the real part in the simulated results.

Fig. 7(a) shows two groups of dispersion curves for the modes in the PC-NCF with different bend radii: the upper group is for the mode $\mathrm{HE}_{1,1+(35)}$ while the lower group is for the mode $\mathrm{HE}_{1,2+(35)}$. Fig. 8 shows the modal intensity and electric field vector distributions of the $\mathrm{HE}_{1,1+(35)}$ and $\mathrm{HE}_{1,2+(35)}$ modes with $R=\infty$ (infinite, represents the fiber straight state) and $R=$ $66.67 \mathrm{~cm}$ at the wavelengths of $1550 \mathrm{~nm}, 1570 \mathrm{~nm}$, and 1590 $\mathrm{nm}$. The HE mode is two-fold degeneracy of modes $\mathrm{HE}_{\mathrm{a}}$ and $\mathrm{HE}_{\mathrm{b}}$. In a straight PC-NCF the dispersion curves of the modes $\mathrm{HE}_{\mathrm{a}}$ and $\mathrm{HE}_{\mathrm{b}}$ are overlapped as shown in Fig. 7(a). The modal intensity distributions for these modes are identical except for a $\pi / 2$ rotation of the electric vector as shown in Fig. 8 . Under the influence of bending, the electric field directions of the modes $\mathrm{HE}_{\mathrm{a}}$ and $\mathrm{HE}_{\mathrm{b}}$ will change to be odd or even along the bending direction, as shown by the $\mathrm{HE}_{1, \mathrm{n}+(35) \mathrm{a}}$ and $\mathrm{HE}_{1, \mathrm{n}+(35) \mathrm{b}}(\mathrm{n}=1$ or 2$)$ with $R=66.67 \mathrm{~cm}$. The bending breaks the degeneracy between the $\mathrm{HE}_{1, \mathrm{n}+(35) \mathrm{a}}$ and $\mathrm{HE}_{1, \mathrm{n}+(35) \mathrm{b}}$ modes as evident by the separated dispersion curves, which is obvious near the resonant bands while less obvious far from them. The $n_{\text {eff }}$ of modes increases as the bend radius decreases, where the increasing amplitude is higher near the resonant bands (around $1550 \mathrm{~nm}$ and $1590 \mathrm{~nm}$ ) compared to those in the anti-resonant band (around $1570 \mathrm{~nm}$ ).

Fiber bending skews the modal intensity distribution toward the outer edge of the fiber bend, with the result that light leaks from the core region into the cladding region at the bend, as shown by the modes $\mathrm{HE}_{1, \mathrm{n}+(35)}(\mathrm{n}=1$ or 2$)$ with $R=66.67 \mathrm{~cm}$ in Fig. 8. The modal field distortions are obvious at wavelengths of $1550 \mathrm{~nm}$ and $1590 \mathrm{~nm}$ around the resonant bands while less obvious at the wavelength of $1570 \mathrm{~nm}$ in the middle of the anti-resonant band, which is consistent with the change of the dispersion curves shown in Fig. 7(a). The modes in the bending states show strong hybridization with the cladding modes. For example, the azimuthal field distribution in the cladding region of the $\mathrm{HE}_{1,2+(35)}$ mode at the wavelength of $1590 \mathrm{~nm}$ with $R=$ $66.67 \mathrm{~cm}$ has a pattern with six radial dark lines. The characteristics of the intensity distribution in the cladding region are similar with the cladding mode $\mathrm{EH}_{6,35}$ shown in Fig. 3(b). The hybridization phenomenon was also found in the hypocycloid-shaped hollow-core photonic crystal fiber [37], which indicates the resonant coupling between the core and the cladding modes.

To quantify the change in modal intensity distribution and to compare the responses of different order modes to fiber bending, the fractional power in the fiber cladding region is calculated numerically using the following expression:

$$
\Gamma_{\mathrm{cl}}=\frac{\iint P_{\mathrm{z}} \cdot d s_{\mathrm{cl}}}{\iint P_{\mathrm{z}} \cdot d s_{\mathrm{cl}}+\iint P_{\mathrm{z}} \cdot d s_{\mathrm{co}}}
$$

where $s_{\mathrm{cl}}$ and $s_{\mathrm{co}}$ represent the cross-section areas of the fiber cladding and core, respectively. $P_{z}$ is the $\mathrm{z}$ component of Poynting vector, calculated by: 

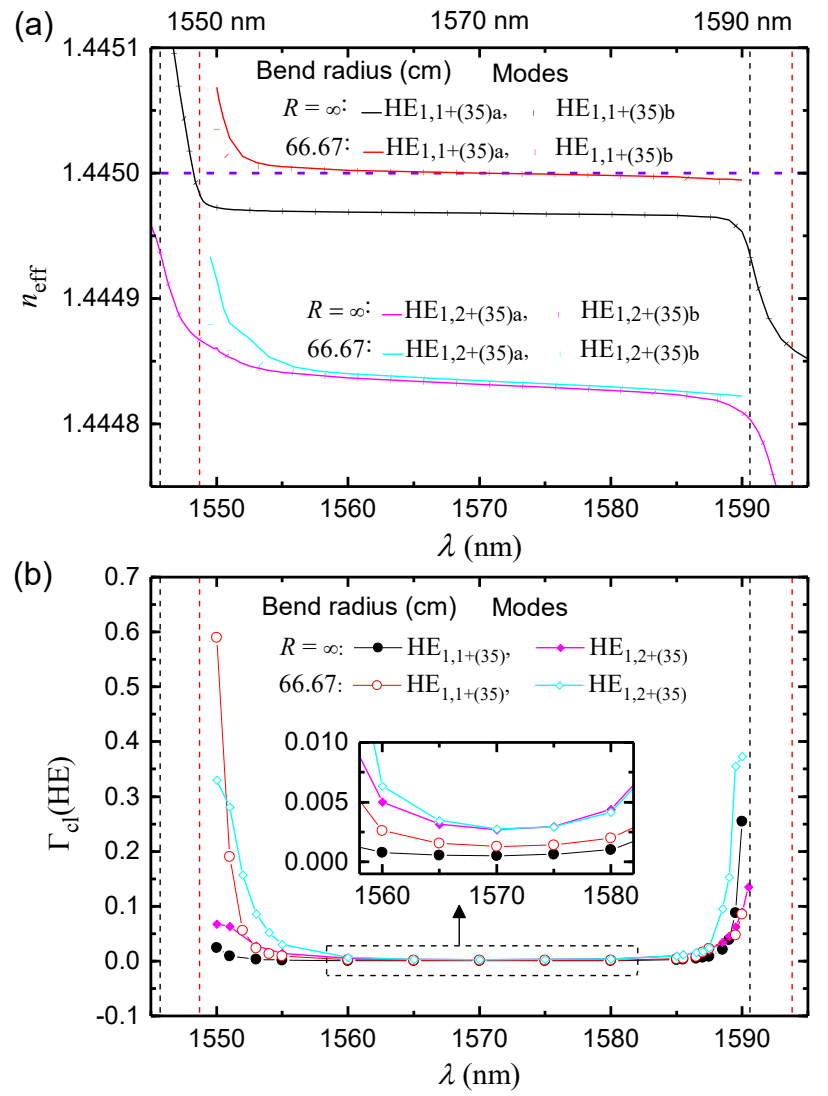

Fig. 7. (a) Dispersion curves ( $n_{\text {eff }}$ vs. $\lambda$ ) and (b) the fractional power in the cladding of $\mathrm{HE}_{1,1+(35)}$ and $\mathrm{HE}_{1,2+(35)}$ modes with the bend radius $R=\infty$ and $R=$ $66.67 \mathrm{~cm}$. The inset of (b) is an enlarged figure of the part indicated by the black dashed rectangle. $\mathrm{HE}_{1, \mathrm{n}+(35)}(\mathrm{n}=1$ or 2$)$ is a two-fold degeneracy mode of $\mathrm{HE}_{1, \mathrm{n}+(35) \mathrm{a}}$ and $\mathrm{HE}_{1, \mathrm{n}+(35) \mathrm{b}}$. The black and red dashed vertical lines indicate the resonant bands.

$$
P_{\mathrm{z}}=\frac{1}{2} \operatorname{Re}\left(H_{y}^{*} E_{x}-H_{x}^{*} E_{y}\right)
$$

Since the HE is a two-fold degeneracy mode, the fractional power in the cladding is the average of the modes $\mathrm{HE}_{\mathrm{a}}$ and $\mathrm{HE}_{\mathrm{b}}$, as follows:

$$
\Gamma_{\mathrm{cl}}(\mathrm{HE})=\frac{1}{2}\left(\Gamma_{\mathrm{cl}}\left(\mathrm{HE}_{a}\right)+\Gamma_{\mathrm{cl}}\left(\mathrm{HE}_{b}\right)\right)
$$

Fig. 7(b) shows that the fractional power in the cladding $\Gamma_{\mathrm{cl}}(\mathrm{HE})$ for the $\mathrm{HE}_{1,1+(35)}$ and $\mathrm{HE}_{1,2+(35)}$ modes, with $R=\infty$ and $R$ $=66.67 \mathrm{~cm}$. The $\Gamma_{\mathrm{cl}}(\mathrm{HE})$ of modes in the middle region of the anti-resonant band is smaller than that near the resonant bands indicated by the vertical black and red dashed lines, for the straight PC-NCF with $R=\infty$. The modes near the resonant bands are more sensitive to the fiber bending than those in the anti-resonant bands, where the $\Gamma_{\mathrm{cl}}(\mathrm{HE})$ of both the $\mathrm{HE}_{1,1+(35)}$ and $\mathrm{HE}_{1,2+(35)}$ modes increases significantly near the resonant bands. The enlarged insert figure in Fig. 7(b) shows that the $\Gamma_{\mathrm{cl}}(\mathrm{HE})$ of the high-order mode $\mathrm{HE}_{1,2+(35)}$ is larger than that of the low-order mode $\mathrm{HE}_{1,1+(35)}$ in the anti-resonant bands, which means that the confinement loss of the former is larger than that of the latter. (a)

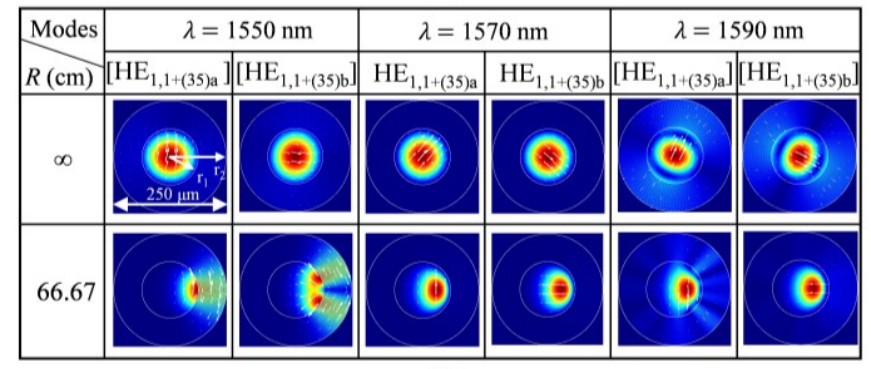

(b)

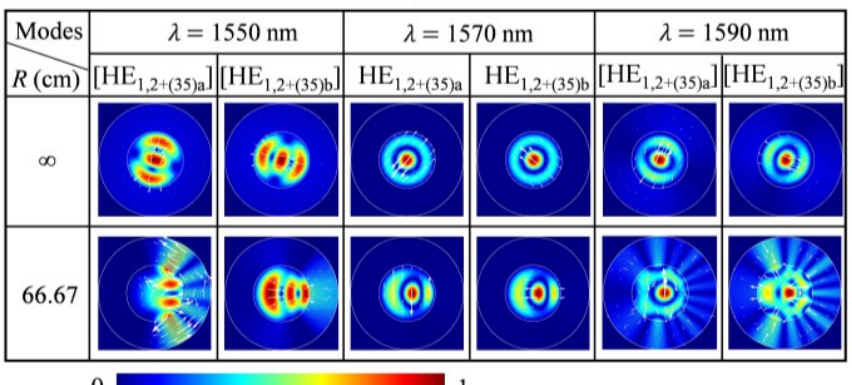

Fig. 8. The modal intensity and electric field vector distributions of $\mathrm{HE}_{1,1+(35)}$ and $\mathrm{HE}_{1,2+(35)}$ modes under different bending states as indicated.

\section{E. Measured transmission spectra of the bent PC-NCFs}

Using the previously described experimental setup in Fig. 1(c), Fig. 9 shows the measured transmission spectra for SMF-PC-NCF-SMF with $L=5.8 \mathrm{~cm}$ and $75.8 \mathrm{~cm}$ under different bending states. The spectrum of the structure with $L=$ $5.8 \mathrm{~cm}$ and $R=\infty$ shows irregular interference dips in Fig. 9(a). The fiber bending changes the spectrum with irregular interference dips to a spectrum with periodic transmission dips, although the intensity of the transmission windows is inconsistent, as one can see for the curve with $R=58.41 \mathrm{~cm}$ in Fig. 9(a). The depths of the transmission dips increase (a little) and the intensities of the transmission windows become relatively consistent as the bend radius decreases to $R=28.51$ $\mathrm{cm}$ in Fig. 9(a). The appearance of periodic transmission dips in the spectrum of the bent PC-NCF with the short length $L$ can be explained in two steps. Firstly, fiber bending shifts the modal intensity distribution from the silica core region into the polymer cladding region, which causes an increase of loss in all bands since the material absorption in the polymer is higher than that in the silica. Secondly, the fractional power in the cladding is larger in the resonant bands than in the anti-resonant bands, which indicates that there is more loss in the former bands than in the latter bands. Therefore, fiber bending can lead to the formation of the periodic transmission dips and an increase of their depths in the PC-NCFs with a short length.

Similarly, the depth of the transmission dips increases as the bend radius decreases for the PC-NCF with a large length of $L=75.8 \mathrm{~cm}$, as shown in Fig. 9(b). Under the similar bend radii, the depth of the transmission dips for the PC-NCF with $L=5.8$ $\mathrm{cm}$ (around $5 \mathrm{~dB}, R=58.41 \mathrm{~cm}$, in Fig. 9(a)) is smaller than that for the PC-NCF with $L=75.8 \mathrm{~cm}$ (around $20 \mathrm{~dB}, R=55.68 \mathrm{~cm}$, in Fig. 9(b)). The larger depth of the transmission dips for the longer PC-NCF is caused by a larger propagation loss, provided 

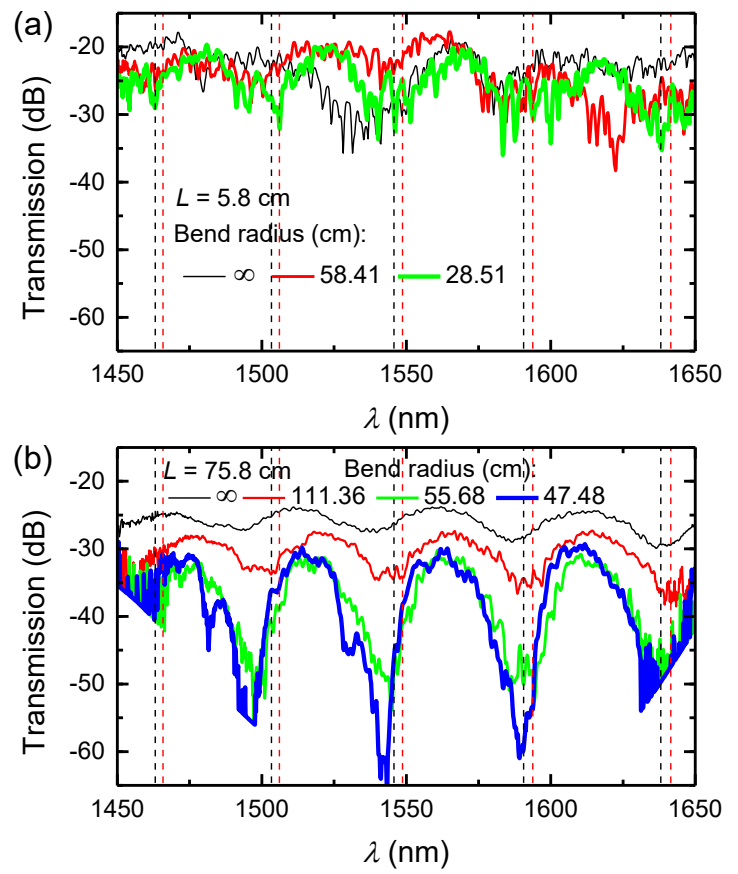

Fig. 9. Measured transmission spectra of the hetero-structure SMF-PC-NCF-SMF with (a) $L=5.8 \mathrm{~cm}$ and (a) $L=75.8 \mathrm{~cm}$, under different bending states as indicated. The black and red dashed vertical lines indicate the resonant bands.

that the confinement losses (the fractional power in the cladding) of the modes are similar for both the long and short PC-NCFs. The intensity of the transmission windows of the PC-NCF with $L=75.8 \mathrm{~cm}$ decreases strongly as the bend radius decreases from $R=\infty$ to $R=113.36 \mathrm{~cm}$ as shown in Fig. 9(b). The situation is different for the PC-NCF with $L=5.8 \mathrm{~cm}$ as shown in Fig. 9(a), where the intensity of the transmission window does not decrease noticeably within the studied range of bend radius. The decreased intensity of the transmission windows is related to the attenuation of the (especially for the higher order) anti-resonant core modes. The fractional power in the cladding of the high-order modes is larger than that of the low-order modes, as discussed in section III-D.

The consequence of introducing a fiber bend is that compared to the low-order modes, the high order modes are more easily affected by bending, which shifts the modal field into the fiber cladding. Therefore, the higher order modes propagating in a bent $\mathrm{PC}-\mathrm{NCF}$ experience higher loss and some of them will fade with the decrease of the bend radius, leading to the decrease of the intensity of the transmission windows. The shorter the propagation length the lower the attenuation of the anti-resonant core modes, therefore, there is no significant reduction in the intensity of the transmission windows for the short PC-NCF within a certain range of bend radius.

The depth of the transmission loss dip around $1550 \mathrm{~nm}$ for the PC-NCF with $L=75.8 \mathrm{~cm}$ increases over $31 \mathrm{~dB}$ from 3.54 $\mathrm{dB}$ to $34.65 \mathrm{~dB}$ as the bend radius decreases from $R=\infty$ to $R=$ $47.48 \mathrm{~cm}$ (the bending curvature increases from 0 to $2.106 \mathrm{~m}^{-1}$ ). The average change rate of the depth in the studied range is up to $14.77 \mathrm{~dB} / \mathrm{m}^{-1}$.

\section{DISCUSSION}

A PC-NCF has been studied as an example of the depressed core fiber in this work. Compared to the more frequently studied anti-resonant guiding hollow-core fibers, whose core modes have a leaky nature $[26,38,39]$, the depressed core fiber has the non-leaky anti-resonant core modes. The energy of the core modes in the hollow-core fibers tends to leak out from both the core and the cladding regions at the resonant bands while that of the depressed core fibers are totally reflected back at the outer boundary of the cladding region although they can leak from the core region. Therefore, the hollow-core fibers generally show periodic transmission loss dips $[26,39,40]$ while the depressed core fiber with multiple non-leaky core modes are prone to showing multimode interference characteristics of the transmission spectrum. However, the appearance of the periodic transmission dips and windows in the long or bent PC-NCFs demonstrated in this work reflect the anti-resonant nature of the depressed core fibers.

The LMR fiber structures are similar with the PC-NCFs, although the coating materials (i.e. ITO in Ref. [20]) of the former are strongly lossy and usually have a complex refractive index. The generation of the loss dips is related to the resonant coupling of the core modes and cladding modes in both the PC-NCF and the LMR fiber structures. The resonant coupling in both structures causes similar changes of the modal field distributions in the resonant bands, where the modal energy is evenly distributed in both the core and the cladding regions [22]. If the high-index coating in the LMR structure is treated as a Fabry-Perot resonator in the ARROW model, it is easy to understand the general rules of the LMR with the help of Eq. (1). For example, the resonant wavelengths are dependent on the thickness and the refractive index of the high-index layer (if other parameters are constant), which is near to the cutoff of the TE and TM modes in the high-index layer [20]. In addition, the Eq. (1) also helps to explain the generation of the multiple LMRs with the thick coating and the excitation of the LMR by both TE and TM light [20]. It should be note that the coating materials in LMR fiber structures are usually much more lossy compared to the acrylate polymer in $\mathrm{PC}-\mathrm{NCF}$, therefore the loss dips can be formed with a much shorter length for the coating (for example the ITO-coated region is $4 \mathrm{~cm}$ in [20]).

The sensing mechanism for the PC-NCF is similar to that for the LMR structure and relies on the change of the thickness and refractive index contrast of the high-index coating and the intensity of the evanescent wave generated at the outer boundary of the coating layer $[3,5,6]$. Based on the theoretical analysis in this work, several methods of reducing the length of the PC-NCF in practical devices such as curvature sensors and optical filters exist. As discussed above, one method of achieving periodic transmission dips in the PC-NCF with a short length $L$ is to introduce bending of the fiber, which leads to an increase of the fractional power in the polymer coating and consequently to an increase of losses in the coating. In a similar fashion, reducing the mode confinement by tapering the silica fiber can increase the fractional power in the cladding, which may allow one to shorten the length of the device. 
Increasing the losses within the polymer coating may also allow to reduce the length of the fiber. In addition, our previous experiments indicated that bending of any short part of the PC-NCF while keeping the rest of the structure straight also allows for the formation of periodic transmission dips, which could reduce the length of the device.

\section{CONCLUSION}

Light propagation in PC-NCFs was studied experimentally and theoretically. The periodic loss dips were found in the transmission spectra of the PC-NCFs with a longer length or under bending states. The wavelength positions of the transmission dips can be predicted based on the ARROW model, with the knowledge of the anti-resonant and inhibited coupling guiding mechanisms. The fractional power in the fiber cladding (polymer coating) for the modes in the resonant bands is higher than that for the modes in the anti-resonant bands, and the fiber bending can enhance this difference. Therefore, the modes in the resonant bands experience higher attenuation due to the material absorption within the polymer coating, which suppresses the multimode interference and displays anti-resonant characteristics. Our results indicate that the PC-NCFs or the depressed core fibers can be used in many devices including bend/curvature sensors and tunable loss filters. Our analysis, regardless of the specific refractive index dispersion properties of the high-index fiber layers, may contribute to a better understanding of the resonant phenomenon in fiber optics such as the LMR in lossy film coated fiber structures.

\section{APPENDIX}

The multiple reflections of one beam incident on an asymmetric planar waveguide are considered in this section. The asymmetric planar waveguide consists of three regions with refractive indices of $n_{1}, n_{2}$ and $n_{3}\left(n_{2}>n_{1}>n_{3}\right)$ as indicated in Fig. 10(a). The angle of incidence is $\theta_{1}$ within $n_{1}>n_{1} \sin \theta_{1}>$ $n_{3}$, which ensures that Fresnel's refraction and reflection at the interface between regions I and II is as shown in Fig. 10(b) while total reflection at the interface between regions II and III is as shown in Fig. 10(c).

The Fresnel's (intrinsic) transmission $(t)$ and reflection $(r)$ coefficients for TE beams are [40]:

$$
r_{\perp i j}=\frac{n_{i} \cos \theta_{i}-n_{j} \cos \theta_{j}}{n_{i} \cos \theta_{i}+n_{j} \cos \theta_{j}}, t_{\perp i j}=\frac{2 n_{i} \cos \theta_{i}}{n_{i} \cos \theta_{i}+n_{j} \cos \theta_{j}}
$$

and for TM beams are [40]:

$$
r_{\| i j}=\frac{n_{j} \cos \theta_{i}-n_{i} \cos \theta_{j}}{n_{j} \cos \theta_{i}+n_{i} \cos \theta_{j}}, t_{\| i j}=\frac{2 n_{i} \cos \theta_{i}}{n_{j} \cos \theta_{i}+n_{i} \cos \theta_{j}}
$$

where indices $i=1(2)$ and $j=2(1)$, denote the incident region and transmission region, respectively.

The intrinsic reflection coefficient $(r)$ and the phase change $(\phi)$ in the total reflection case are as follows. For the TE beams: (a)

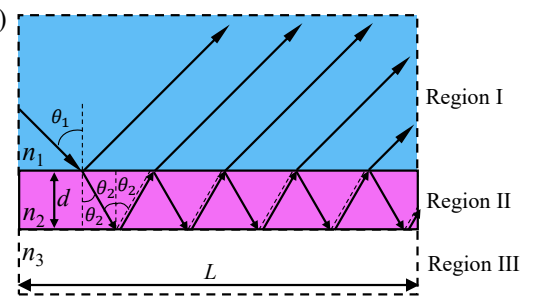

$\left(n_{2}>n_{1}>n_{3}\right)$
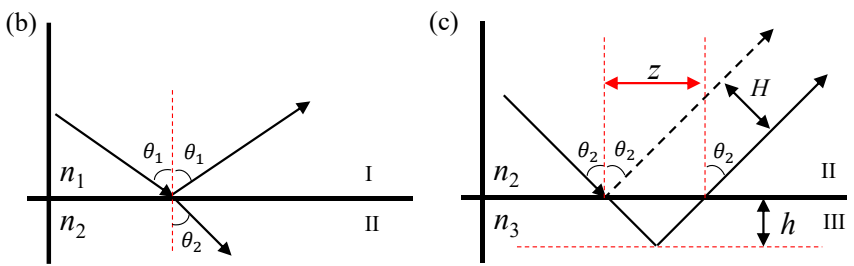

Fig. 10. (a) Ray trajectory of a beam with angle of incidence $\theta_{1}\left(n_{1}>n_{1} \sin \theta_{1}>\right.$ $n_{3}$ ) impinging on the interface of an asymmetric waveguide. (b) Fresnel's refraction and reflection at the interface between regions I and II. (c) Total reflection at the interface between regions II and III, where ' $z$ ', ' $H$ ', and ' $h$ ' denote the Goos-Hänchen shift, the deviation of the reflection light, and the penetration depth of the evanescent wave, respectively.

$$
r_{\perp i j}=e^{i 2 \phi_{\perp}}, \phi_{\perp}=\tan ^{-1}\left(\frac{\sqrt{\sin ^{2} \theta_{i}-n_{j}^{2} / n_{i}^{2}}}{\cos \theta_{i}}\right)
$$

For the TM beams:

$$
r_{\| i j}=e^{i 2 \phi_{\|}}, \phi_{\|}=\tan ^{-1}\left(\frac{n_{i}^{2}}{n_{j}^{2}} \frac{\sqrt{\sin ^{2} \theta_{i}-n_{j}^{2} / n_{i}^{2}}}{\cos \theta_{i}}\right)
$$

where index $i=2$ and $\mathrm{j}=3$ denote regions II and III, respectively.

The total amplitude of multiple reflections for TE (or TM) beams in the region II can be calculated as:

$$
\begin{aligned}
& A^{(r)} \\
& =\left[r_{12}+t_{12} r_{23} t_{21} e^{i \delta}\right. \\
& +t_{12} r_{23}\left(r_{21} r_{23}\right) t_{21} e^{i 2 \delta}+t_{12} r_{23}\left(r_{21} r_{23}\right)^{2} t_{21} e^{i 3 \delta} \\
& \left.+\cdots+t_{12} r_{23}\left(r_{21} r_{23}\right)^{p-2} t_{21} e^{i(p-1) \delta}\right] A^{(i)} \\
& =\left\{r_{12}+t_{12} r_{23} t_{21}\left[\frac{1-\left(r_{21} r_{23} e^{i \delta}\right)^{p-1}}{1-r_{21} r_{23} e^{i \delta}}\right] e^{i \delta}\right\} A^{(i)}
\end{aligned}
$$

where $\delta$ is the phase difference between the two adjacent reflected beams in region I: $\delta=\frac{4 \pi n_{2} d \cos \theta_{2}}{\lambda} . p$ is number of reflected beams: $p=\frac{L}{2 d \tan \theta_{2}} . A^{(i)}$ is the amplitude of the incident beam. Given $d=125 \mu \mathrm{m}, n_{1}=1.445, n_{2}=1.51$, and a waveguide length $L>0.5 \mathrm{~cm}$, the $p$ is large enough for the total reflected amplitude to be approximated as:

$$
A^{(r)}=\left(r_{12}+\frac{t_{12} r_{23} t_{21}}{1-r_{21} r_{23} e^{i \delta}} e^{i \delta}\right) A^{(i)}
$$

And the ratio of reflection intensity can be calculated as: 


$$
R=\frac{A^{(r)} A^{(r) *}}{A^{(i)} A^{(i) *}}=\left|r_{12}+\frac{t_{12} r_{23} t_{21}}{1-r_{21} r_{23} e^{i \delta}} e^{i \delta}\right|^{2}
$$

Substituting the intrinsic reflection and refraction coefficient for the TE (or TM) beams calculated by Eqs. (12)-(15) into Eqs. (17) and (18), the ratio of reflection intensity of the TE and TM beams are calculated respectively as $R_{\perp}$ and $R_{\|}$. The ratio of reflection intensity of an unpolarized incident beam is the average of $R_{\perp}$ and $R_{\|}$thus:

$$
\bar{R}=\frac{R_{\perp}+R_{\|}}{2}
$$

\section{REFERENCES}

[1] X.-d. Wang and S. W. Otto, "Fiber-optic chemical sensors and biosensors (2013-2015)," Analytical Chem., vol. 88, no. 1, pp. 203-227, 2015.

[2] C. Caucheteur, T. Guo, and J. Albert, "Review of plasmonic fiber optic biochemical sensors: Improving the limit of detection," Anal. Bioanal. Chem., vol. 407, no. 14. pp. 3883-3897, 2015.

[3] I. D. Villar et al., "Optical sensors based on lossy-mode resonances," Sens. Actuators B Chem., vol. 240, pp. 174-185, Mar. 2017.

[4] K. M. Tripathi, T. Y Kim, D. Losic, and T. T. Tung, "Recent advances in engineered graphene and composites for detection of volatile organic compounds (VOCs) and non-invasive diseases diagnosis," Carbon, vol. 110, pp. $97-129,2016$.

[5] A. Ozcariz, D. A. Piña-Azamar, C. R. Zamarreño, R. Dominguez, and F. J. Arregui, "Aluminum doped zinc oxide (AZO) coated optical fiber LMR refractometers - An experimental demonstration," Sensors and Actuators B: Chemical, vol. 281, pp. 698-704, 2019.

[6] M. Śmietana, et al., "Simultaneous optical and electrochemical label-free biosensing with ITO-coated lossy-mode resonance sensor," Biosensors and Bioelectronics, vol. 154, pp. 112050, 2020.

[7] Y. Wang et al., "Fiber optic relative humidity sensor based on the tilted fiber Bragg grating coated with graphene oxide," Appl. Phys. Lett., vol. 109, no. 3, Jul. 2016, Art. no. 031107.

[8] Q. Wu, Y. Semenova, J. Mathew, P. Wang, and G. Farrell, "Humidity sensor based on a single-mode hetero-core fiber structure," Opt. Let., vol. 36, no. 10, pp. 1752-1754, 2011

[9] D. Liu et al., "High sensitivity sol-gel silica coated optical fiber sensor for detection of ammonia in water," Opt. Express, vol. 24, pp. 24179-24187, 2016

[10] J. Ascorbe, J. M. Corres, F. J. Arregui, and I. R. Mat' ias, "Optical fiber current transducer using lossy mode resonances for high voltage networks," J. Lightw. Technol., vol. 33, no. 12, pp. 2504-2510, Jun. 2015

[11] J. M. Corres, J. Ascorbe, F. J. Arregui, and I. R. Matias, "Tunable electro-optic wavelength filter based on lossy-guided mode resonances," Opt. Express, vol. 21, no. 25, pp. 31668-31677, 2013

[12] Y. Zhang, H. Li, C. Dai, L. Xu, C. Gu, W. Chen, Y. Zhu, P. Yao, and Q. Zhan, "All-fiber high-order mode laser using a metal-clad transverse mode filter," Opt. Express, vol. 26, no. 23, pp. 29679-29686, 2018.

[13] H. Zhang, N. Healy, L. Shen, C. C. Huang, D. W. Hewak, and A. C. Peacock, "Enhanced all-optical modulation in a graphene-coated fibre with low insertion loss," Sci. Rep., vol. 6, 2016, Art. no. 23512.

[14] I. Del Villar, I. R. Matias, F. J. Arregui, and P. Lalanne, "Optimization of sensitivity in long period fiber gratings with overlay deposition," Opt. Express, vol. 13, no. 1, pp. 56-69, Jan. 2005.

[15] A. Cusano, A. Iadicicco, P. Pilla, L. Contessa, S. Campopiano, A. Cutolo, and M. Giordano, "Mode transition in high refractive index coated long period gratings," Opt. Exp., vol. 14, no. 1, Jan. 2006.

[16] L.-L. Xue and L. Yang, "Sensitivity enhancement of RI sensor based on SMS fiber structure with high refractive index overlay," J. Lightw. Technol., vol. 30, no. 10, pp. 1463-1469, May 2012.

[17] N. D Rees, S. W James, R. P. Tatam, and G. J Ashwell, "Optical fibre long period gratings with Langmuir Blodgett thin film overlays," Opt. Lett., vol. 27, no. 9, pp. 686-688, May 2002.

[18] A. Socorro, I. Del Villar, J. Corres, F. Arregui, and I. Matias, "Sensitivity enhancement in a multimode interference-based SMS fibre structure coated with a thin-film: Theoretical and experimental study," Sens. Actuators B, Chem., vol. 190, pp. 363-369, 2014.
[19] C. R. Zamarreno, M. Hernaez, I. Del Villar, I. R. Matias, and F. J. Arregui, "Optical fiber $\mathrm{pH}$ sensor based on lossy-mode resonances by means of thin polymeric coatings," Sens. Actuators B Chem., vol. 155, no. 1, pp. 290-297, Jul. 2011.

[20] I. Del Villar, C. R. Zamarreno, M. Hernaez, F. J. Arregui, and I. R. Matias, "Lossy mode resonance generation with indium tin oxide coated optical fibers for sensing applications," J. Lightw. Technol., vol. 28, pp. 111-117,2010.

[21] J. M. Corres, I. D. Villar, F. J. Arregui, and I. R. Matias. "Analysis of lossy mode resonances on thin-film coated cladding removed plastic fiber." Opt. let., vol. 40, no. 21, pp. 4867-4870, 2015.

[22] I. D. Villar, I. R. Matias, F. J. Arregui, and M. Achaerandio, "Nanodeposition of materials with complex refractive index in long-period fiber gratings," J. Lightw. Technol., vol. 23, no. 23, pp. 4192-4199, Dec. 2005.

[23] W. M. Zhao, Q. Wang, J. Y. Jing, and X. Z. Wang, "Discoveries and explorations of mode splitting phenomenon in lossy dielectric waveguide," Plasmonics, vol. 15, no. 2, pp. 481-487, 2020.

[24] C. Tsao, Optical Fibre Waveguide Analysis. London, U.K.: Oxford Univ. Press, 1992.

[25] X. Lian, G. Farrell, Q. Wu, W. Han, C. Shen, Y. Ma, and Yuliya Semenova. "Anti-resonance, inhibited coupling and mode transition in depressed core fibers." Opt. express, vol. 28, no. 11, pp. 16526-16541, May 2020.

[26] M. Duguay, Y. Kokubun, T. Koch, and L. Pfeiffer, "Antiresonant reflecting optical wave-guides in $\mathrm{SiO}_{2}$-Si multilayer structures," Appl. Phys. Lett., vol. 49, no. 1, pp. 13-15, July 7, 1986.

[27] N. Sultanova, S. Kasarova, and I. Nikolov, "Dispersion properties of optical polymers," Acta. Phys. Pol. A, vol. 116, no. 4, pp. 585-587, Mar. 2009.

[28] T. Eiselt, J. Preinfalk, U. Gleißner, U. Lemmer, and T. Hanemann. "Development and characterization of adjustable refractive index scattering epoxy acrylate polymer layers." In Light Manipulating Organic Materials and Devices III, vol. 9939, p. 99390Q. International Society for Optics and Photonics, 2016.

[29] K. Tian, G. Farrell, E. Lewis, X. Wang, H. Liang, and P. Wang, "A high sensitivity temperature sensor based on balloon-shaped bent SMF structure with its original polymer coating," Meas. Sci. Technol., vol. 29, no. 8, pp. 085104, 2018.

[30] Y. P. Wang, et al., "Intensity measurement bend sensors based on periodically tapered soft glass fibers," Opt. Lett., vol. 36, no. 4, pp. 558-560, Feb. 2011.

[31] D. Marcuse, Theory of Dielectric Optical Waveguides. Elsevier, 2013.

[32] W. S. Mohammed, A. Mehta, and E. G. Johnson, "Wavelength tunable fiber lens based on multimode interference," J. Lightw. Technol., vol. 22, no. 2, p. 469, Feb. 2004.

[33] Q. Wang, G. Farrell, and W. Yan, "Investigation on single-mode-multimodesingle-mode fiber structure," J. Lightw. Technol., vol. 26, no. 5, pp. 512-519, Mar. 2008.

[34] X. Lian, Q. Wu, G. Farrell, C. Shen, Y. Ma, and Y. Semenova, "Discrete self-imaging in small-core optical fiber interferometers." J. Lightw. Technol., vol. 37, no. 9, pp. 1873-1884, 2019.

[35] H. Ma, A. K.-Y. Jen, and L. R. Dalton, "Polymer-based optical waveguides: Materials, processing, and devices," Adv. Mater., vol. 14, no. 19, pp. 1339$1365,2002$.

[36] M. Heiblum and J. Harris, "Analysis of curved optical waveguides by conformal transformation," IEEE J. Quantum Electron., vol. QE-11, no. 2, pp. 75-83, Feb. 1975.

[37] B. Debord, M. Alharbi, T. Bradley, C. Fourcade-Dutin, Y. Y. Wang, L. Vincetti, F. Gérôme, and F. Benabid, "Hypocycloid-shaped hollow-core photonic crystal fiber Part I: Arc curvature effect on confinement loss," Opt. Exp., vol. 21, no. 23, pp. 28597-28608, 2013.

[38] J. L. Archambault, R. J. Black, S. Lacroix, and J. Bures, "Loss calculations for antiresonant waveguides," J. Lightw. Technol., vol. 11, no. 3, pp. 416-423, Mar. 1993.

[39] N. M. Litchinitser, A. K. Abeeluck, C. Headley, and B. J. Eggleton, "Antiresonant reflecting photonic crystal optical waveguides," Opt. Lett, vol. 27, no. 18, pp. 1592-1594, 2002.

[40] M. Born, and E. Wolf, Principles of Optics: Electromagnetic Theory of Propagation, Interference and Diffraction of Light. Elsevier, 2013. 